\title{
Metabolomic changes in animal models of depression: a
} systematic analysis

\author{
Juncai Pu ${ }^{1,2,4}$, Yiyun $\mathrm{Liu}^{1,4}$, Siwen Gui ${ }^{1,4}$, Lu Tian ${ }^{1}$, Yue $\mathrm{Yu}^{3}$, Xuemian Song ${ }^{1}$, Xiaogang Zhong ${ }^{1}$, Xiaopeng Chen ${ }^{1,2}$, Weiyi Chen ${ }^{1,2}$, \\ Peng Zheng ${ }^{1,2}$, Hanping Zhang ${ }^{1,2}$, Xue Gong ${ }^{1,2}$, Lanxiang Liu ${ }^{1}$, Jing Wu ${ }^{1}$, Haiyang Wang ${ }^{1}$ and Peng Xie (iD ${ }^{1,2}{ }^{凶}$
}

(c) The Author(s) 2021

\begin{abstract}
Extensive research has been carried out on the metabolomic changes in animal models of depression; however, there is no general agreement about which metabolites exhibit constant changes. Therefore, the aim of this study was to identify consistently altered metabolites in large-scale metabolomics studies of depression models. We performed vote counting analyses to identify consistently upregulated or downregulated metabolites in the brain, blood, and urine of animal models of depression based on 3743 differential metabolites from 241 animal metabolomics studies. We found that serotonin, dopamine, gamma-aminobutyric acid, norepinephrine, N-acetyl-L-aspartic acid, anandamide, and tryptophan were downregulated in the brain, while kynurenine, myo-inositol, hydroxykynurenine, and the kynurenine to tryptophan ratio were upregulated. Regarding blood metabolites, tryptophan, leucine, tyrosine, valine, trimethylamine $\mathrm{N}$-oxide, proline, oleamide, pyruvic acid, and serotonin were downregulated, while $\mathrm{N}$-acetyl glycoprotein, corticosterone, and glutamine were upregulated. Moreover, citric acid, oxoglutaric acid, proline, tryptophan, creatine, betaine, L-dopa, palmitic acid, and pimelic acid were downregulated, and hippuric acid was upregulated in urine. We also identified consistently altered metabolites in the hippocampus, prefrontal cortex, serum, and plasma. These findings suggested that metabolomic changes in depression models are characterized by decreased neurotransmitter and increased kynurenine metabolite levels in the brain, decreased amino acid and increased corticosterone levels in blood, and imbalanced energy metabolism and microbial metabolites in urine. This study contributes to existing knowledge of metabolomic changes in depression and revealed that the reproducibility of candidate metabolites was inadequate in previous studies.
\end{abstract}

Molecular Psychiatry (2021) 26:7328-7336; https://doi.org/10.1038/s41380-021-01269-w

\section{INTRODUCTION}

Depression is a mental illness that severely impairs the social function of patients. The lifetime prevalence of depression is $\sim 6.8$ to $20.6 \%[1,2]$. In addition, depression severely impairs the quality of life of patients and has become one of the primary diseases leading to mental disability [3]. Despite a large body of evidence supporting the pathophysiology of depression, the underlying molecular mechanisms that mediate its onset remain unclear. Depression has complex and diverse causal factors, a lack of clear pathological alterations or risk genes, and a high degree of heterogeneity in clinical presentation $[4,5]$.

Metabolites are products of upstream gene and protein regulatory networks that are involved in a variety of physiological and pathological conditions [6, 7]. Metabolomics techniques have been rapidly developed in the last two decades [8]. Because of the important physiological role of metabolites, these metabolomics techniques have been widely used to identify biochemical disturbances in diseases [9]. To date, hundreds of metabolomics studies have been conducted to investigate the metabolite alterations in animal models of depression, which expanded the knowledge of the physiopathology of depression [10-12]. Moreover, supplementation with certain metabolites could exert antidepressant effects, suggesting that metabolomics techniques are potential strategies for screening and developing new antidepressants [13-15].

Although extensive research has been carried out on the metabolomic changes in animal models of depression, there are concerns regarding the generalizability of these metabolomics studies, as their results are not consistent [16]. To our knowledge, no previous study has assessed the reproducibility of metabolomics studies, and there is no general agreement about which metabolites show constant changes in animal models of depression. Therefore, the aim of this study was to perform a systematic analysis to identify consistently altered metabolites in animal models of depression by integrating the totality of evidence from these large-scale metabolomics studies. Using a knowledgebasedriven approach, we performed vote counting analyses to identify consistently upregulated or downregulated metabolites in the brain, blood, and urine in animal models of depression.

\section{MATERIALS AND METHODS \\ Data source}

Candidate metabolites were derived from our metabolite database of depression called the Metabolite Network of Depression Database

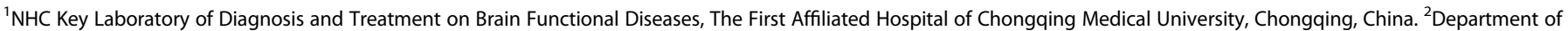

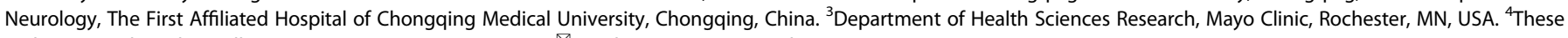
authors contributed equally: Juncai Pu, Yiyun Liu, Siwen Gui. ${ }^{{ }_{\text {email: }}}$ xiepeng@cqmu.edu.cn
} 
(MENDA) (http://menda.cqmu.edu.cn:8080/index.php). Briefly, this database included 464 clinical and preclinical studies that investigated metabolite changes in depression and its treatment using metabolomics and magnetic resonance spectroscopy (MRS) techniques as of March 20 2018 [17]. The search strategy and study selection criteria can be found in a previously published article [17]. We then manually curated 5675 differential metabolites from these studies. The ratios of two metabolites were also included in this database.

In this study, we updated this database up to April 1, 2021, which nearly doubled the numbers of included studies and metabolites. After researching PubMed, the Cochrane Library, Embase, Web of Science, and PsycINFO, we cumulatively reviewed 2177 full-text articles and excluded 1409 studies (Supplementary Data 1). Finally, we included 768 clinical and preclinical metabolomics and MRS studies that investigated metabolic alterations in depression and its treatment. We did not exclude studies that none of the metabolites tested reached significant levels. A total of 12,097 differential metabolites were manually obtained from these studies based on the significance levels reported in the original studies. We did not use uniform statistical criteria for data reanalysis because only 3\% of these studies provided raw data. In addition, we used the Human Metabolome Database (HMDB) [18], PubChem [19], and Kyoto Encyclopedia of Genes and Genomes (KEGG) [20] databases to standardize the synonyms of candidate metabolites.

\section{Data selection}

For further data analysis, we selected curated data according to the following criteria. For study type, we included studies that compared metabolite levels between rodent models of depression and controls and excluded intervention studies that investigated metabolic changes associated with antidepressant treatment. Nonhuman primates and cell studies were excluded. For metabolite detection methods, we included non-targeted and targeted metabolomics studies and excluded in vivo MRS studies. In addition, we excluded metabolites with unknown regulation directions.

\section{Data analysis strategy}

For the quantitative analysis, we used the following analytical strategy. In the main analysis, we analyzed metabolite alterations in brain, blood (plasma and serum), and urine samples. To further analyze the metabolite alterations in different tissues, secondary analyses were performed in the hippocampus, prefrontal cortex, serum, and plasma. Other brain or peripheral tissues were not included because of the small amount of data. In addition, we also explored metabolite alterations in the chronic mild stress (CMS) model, the most commonly used model of depression. The quality of reporting of the included studies was evaluated according to the updated STAIR recommendations [21].

\section{Statistical analysis}

Merging raw data, mean values, or $p$ values are the optimal strategies for merging metabolomics data $[22,23]$. However, a lack of raw data, mean values, or fold changes for each metabolite in most of these metabolomics studies prevented us from conducting a meta-analysis. Alternatively, we used the vote counting process to analyze whether metabolites were consistently upregulated or downregulated across studies. The vote counting method can enrich for candidate molecules that are likely to be confirmed by independent testing [24]. During the vote counting process, each metabolite was noted as "1" or " -1 " when it was reported as significantly upregulated or downregulated in each study, respectively. Where samples were taken from different brain regions in one research, candidate metabolites were considered to originate from independent studies and were counted separately. The vote counting statistic (VCS) for each metabolite was then calculated by summing the scores [25]. Larger or smaller VCS values indicated that more studies reported the metabolite as significantly upregulated or downregulated, respectively.

To analyze whether the upregulation or downregulation of each metabolite was statistically significant, we used a binomial distribution to determine whether the positive significant findings were attributable to chance, with the assumption that each candidate metabolite was upregulated or downregulated in each study with a probability of $50 \%$ [25]. The binomial tests were implemented in R ( $v$ 4.0.4, https://www.rproject.org/) with the function binom.test. The one-tailed $p$ values were calculated for candidate metabolites that were reported in more than three data sets, and the minimum $p$ value for fewer data sets was 0.1 . Differences with $p<0.05$ were considered to be significant.

\section{RESULTS}

\section{Data sets}

Through screening of the MENDA database, 8354 differential metabolites from 527 studies were excluded from the study; see Supplementary Table 1 and Supplementary Data 2 for the reasons for exclusion. A total of 3743 differential metabolites from 241 metabolomics studies in animal models of depression were included in the analysis. The full information of included studies (title, study design, category of depression model, organism, tissue, platform, criteria for depression, sample size, original data availability, and citation) is provided in Supplementary Data 3. The full information of candidate metabolites (metabolite name, HMDB ID, KEGG ID, PubChem ID, comparison group, tissue, organism, category of depression model, platform, and regulation direction) is presented in Supplementary Data 4.

The characteristics of the included studies and metabolites are summarized in Supplementary Table 2. Briefly, brain tissues were used in 151 studies, with 2119 differential metabolites. The hippocampus (95 studies, 1119 differential metabolites) and prefrontal cortex (46 studies, 460 differential metabolites) were the most widely used brain tissues. Regarding peripheral tissues, plasma (53 studies, 415 differential metabolites) was the most commonly used sample type, followed by serum (43 studies, 572 differential metabolites), and urine (37 studies, 637 differential metabolites). Eighty-seven unique metabolites (or metabolite ratios) in the brain, 58 in blood, and 45 in urine were reported as dysregulated in at least four studies (Fig. 1). The CMS model was the most widely used animal model (140 studies, 1955 differential metabolites). The overall quality of reporting is presented in Supplementary Data 5, and few studies have met some of these criteria (e.g., sample size calculation and randomization).

\section{Metabolites altered in the brain}

We assessed which metabolites were consistently dysregulated in the brain. Volcano plots of candidate metabolites in the brain, hippocampus, and prefrontal cortex are presented in Fig. 2A-C. Among the 85 candidate metabolites and two metabolite ratios in the brain, serotonin (VCS $=-28, p<0.001$ ), dopamine (VCS $=-24$, $p<0.001$ ), gamma-aminobutyric acid (GABA, VCS $=-21, p=$ 0.002), norepinephrine (VCS $=-11, \quad p=0.017), \quad N$-acetyl-Laspartic acid (VCS $=-11, p=0.049$ ), anandamide (VCS $=-10$, $p=0.015)$, and tryptophan (VCS $=-10, p=0.049$ ) were downregulated, while kynurenine $(\mathrm{VCS}=19, p<0.001)$, myo-inositol $(\mathrm{VCS}=14, p=0.003)$, hydroxykynurenine (VCS $=11, p=0.002)$, and the kynurenine to tryptophan ratio (VCS $=8, p=0.004)$ were upregulated (Fig. 2D and Supplementary Table 3).

Among the 56 candidate metabolites and two metabolite ratios in the hippocampus, serotonin (VCS $=-17, p<0.001$ ), dopamine $(\mathrm{VCS}=-13, p=0.004)$, and GABA (VCS $=-10, p=0.038)$ were downregulated, and kynurenine (VCS $=11, p=0.002)$ and the kynurenine to tryptophan ratio (VCS $=5, p=0.031$ ) were upregulated (Fig. 2E and Supplementary Table 4). Among the 15 candidate metabolites in the prefrontal cortex, only glutamic acid was dysregulated (VCS $=-8, p=0.019$; Fig. 2F and Supplementary Table 5).

\section{Metabolites altered in blood}

We further analyzed the metabolite alterations in blood. Volcano plots of candidate metabolites in blood, plasma, and serum are presented in Fig. 3A-C. Fifty-seven candidate metabolites and one metabolite ratio in the blood were introduced in the vote counting procedure, and the results revealed that tryptophan $(\mathrm{VCS}=-18, p<0.001)$, leucine (VCS $=-10, p=0.001)$, tyrosine $(\mathrm{VCS}=-9, p=0.002)$, valine $(\mathrm{VCS}=-9, p=0.006)$, trimethylamine $\mathrm{N}$-oxide (VCS $=-9, p=0.011)$, proline $(\mathrm{VCS}=-8, p=0.004)$, oleamide (VCS $=-6, p=0.016)$, pyruvic acid (VCS $=-6, p=$ 0.016 ), and serotonin (VCS $=-5, p=0.031$ ) were downregulated, 

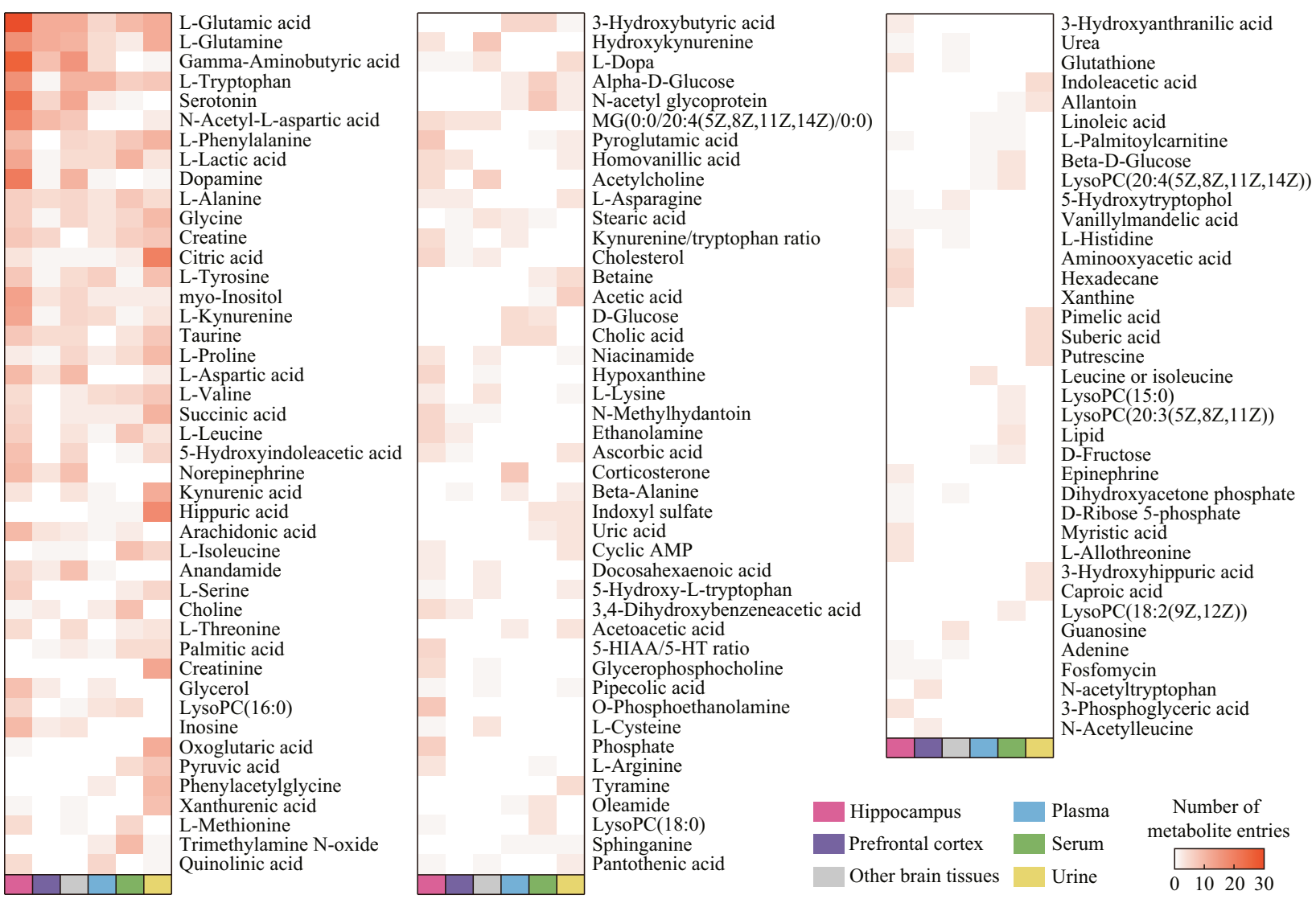

Fig. 1 Distribution of metabolites in brain, blood, and urine samples of depression models. Darker colors in the boxes represent larger values. Only metabolites that were reported as dysregulated in more than three studies for any type of sample are shown. 5-HIAA 5-hydroxyindoleacetic acid, 5-HT serotonin, AMP adenosine monophosphate, LysoPC lysophosphatidylcholine, MG monoacylglycerol.

while N-acetyl glycoprotein (VCS $=11, p<0.001)$, corticosterone $(\mathrm{VCS}=9, p=0.002)$, and glutamine $(\mathrm{VCS}=6, p=0.035)$ were upregulated (Fig. 3D and Supplementary Table 6).

Further analysis showed that three and seven candidate metabolites were consistently dysregulated in plasma and serum samples, respectively (Fig. 3E, F). Tryptophan (VCS $=-11, p<$ $0.001)$, tyrosine (VCS $=-7, p=0.008)$, and corticosterone (VCS $=$ $8, p=0.004$ ) were dysregulated in plasma samples (Supplementary Table 7). Leucine (VCS $=-8, p=0.004$ ), tryptophan (VCS $=$ $-7, p=0.008)$, isoleucine (VCS $=-7, p=0.020)$, valine $(\mathrm{VCS}=-6$, $p=0.016)$, proline (VCS $=-5, p=0.031)$, pyruvic acid (VCS $=-5$, $p=0.031$ ), and $\mathrm{N}$-acetyl glycoprotein (VCS $=8, p=0.004$ ) were dysregulated in serum samples (Supplementary Table 8).

\section{Metabolites altered in urine}

The vote counting procedure revealed that 10 of the 45 candidate metabolites in urine were consistently dysregulated (Fig. 4A). Citric acid (VCS $=-16, p<0.001)$, oxoglutaric acid (VCS $=-10$, $p=0.003)$, proline (VCS $=-8, p=0.011)$, tryptophan $(\mathrm{VCS}=-6$, $p=0.035)$, creatine (VCS $=-6, p=0.035)$, betaine (VCS $=-5, p=$ $0.031)$, L-dopa (VCS $=-5, p=0.031)$, palmitic acid (VCS $=-5, p=$ $0.031)$, and pimelic acid (VCS $=-5, p=0.031)$ were downregulated, and hippuric acid (VCS $=10, p=0.015)$ was upregulated (Fig. 4B and Supplementary Table 9).

\section{Metabolites altered in the CMS model}

To determine whether the vote counting results were influenced by types of models, we investigated the altered metabolites in the CMS model. Six, 13, and five metabolites were consistently dysregulated in brain, blood, and urine samples, respectively (Fig. 5A-C; Supplementary Tables 10-12). All but one of these consistently altered metabolites were shared among the results of all models (Fig. 5D). Regarding other types of models, the vast majority of candidate metabolites were reported in less than three data sets, leaving insufficient data for the vote counting procedure.

\section{DISCUSSION}

This study is the first comprehensive investigation of metabolomic changes in animal models of depression that provided a large evidence synthesis of 241 studies. Consistently dysregulated metabolites in brain, blood, and urine samples across these studies were identified from 3743 differential metabolites using a vote counting approach. We found that decreased neurotransmitter levels and increased kynurenine metabolite levels were the main metabolite signatures in the brains of depression models. Blood was characterized by decreased amino acid concentrations and increased corticosterone levels. We also provided evidence of imbalanced energy metabolism and microbial metabolites in urine.

The main results obtained from this study, contrary to our expectations, revealed the inadequate reproducibility of metabolomics studies. Although nearly 300 metabolomics studies were examined, we only identified approximately 10 consistently altered metabolites in brain, blood, and urine samples. This may have occurred because many factors may influence the identification of differential metabolites. Sample preparation, metabolomics experiments, and data analysis are decisive factors that affect the reproducibility of methods [26, 27]. Indeed, any change in parameter settings in these processes can have substantial effects on the compound identification and quantification results [28]. 


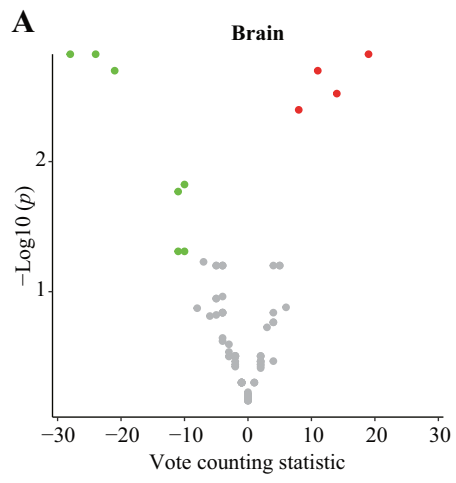

B

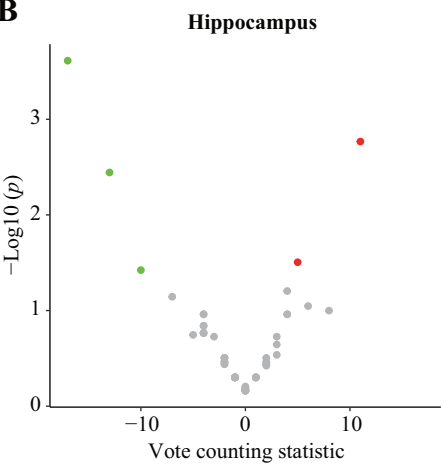

D
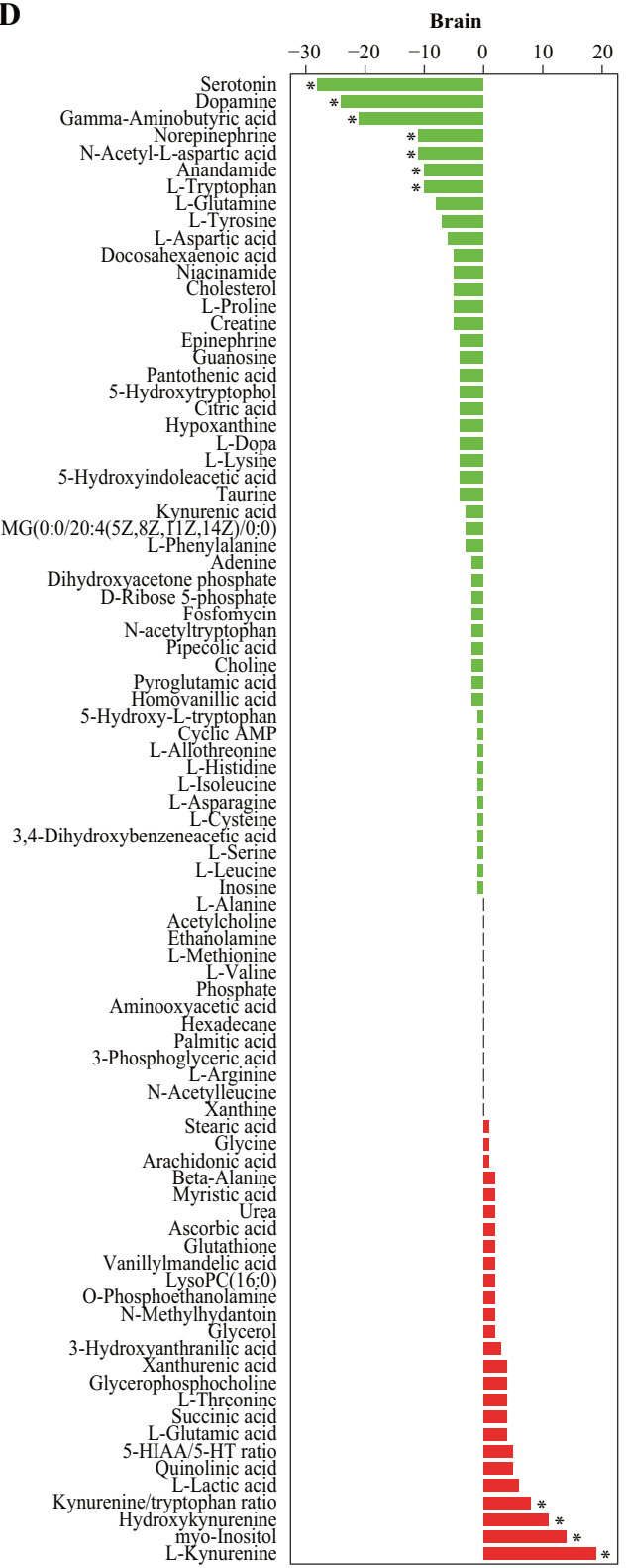

C

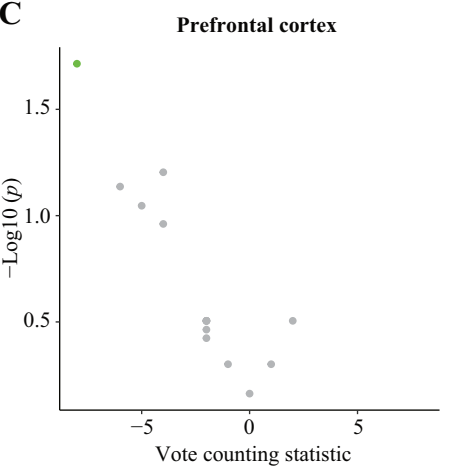

E

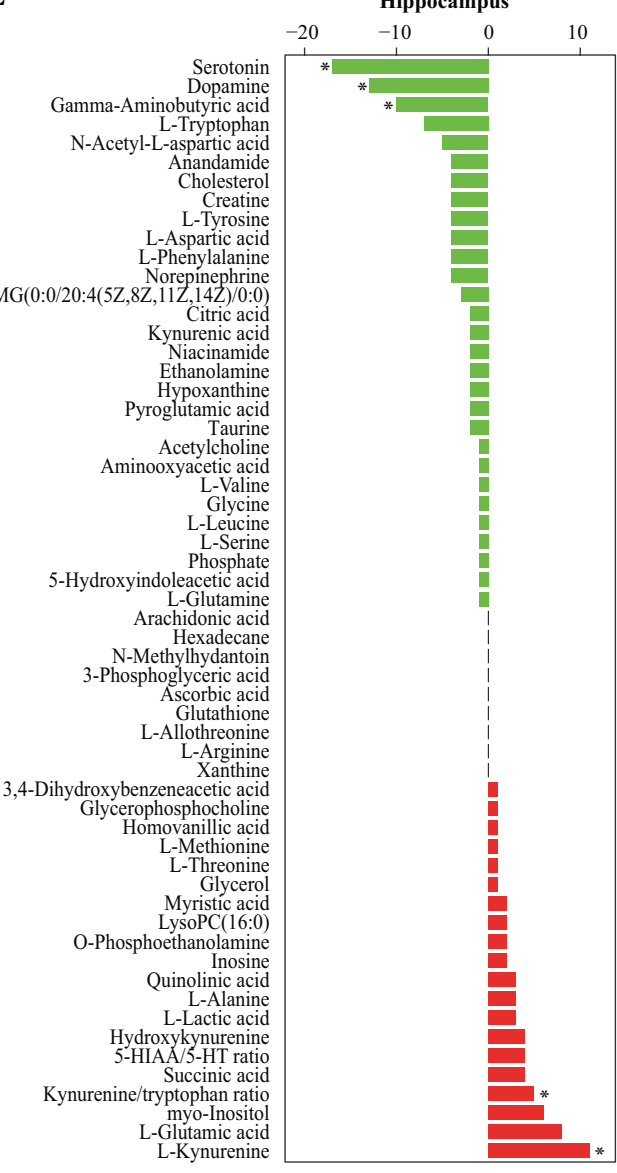

F

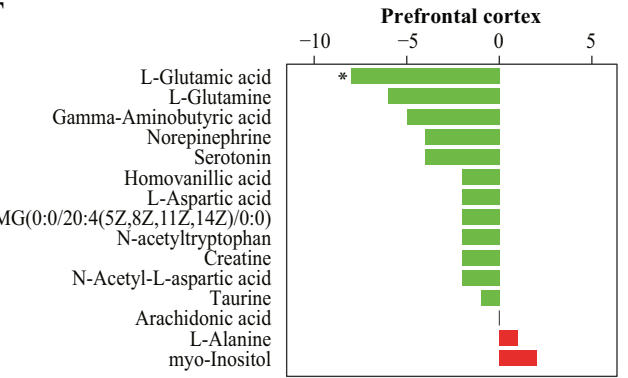

Fig. 2 Vote counting results for the brain. A-C Volcano plots of candidate metabolites in the brain (A), hippocampus (B), and prefrontal cortex (C). Nodes represent candidate metabolites, the $x$-axis shows the vote counting statistic, and the $y$-axis shows the - log 10 ( $p$ value). Red, green, and gray nodes denote consistently upregulated, downregulated, or unaltered metabolites, respectively. D-F Bar plots of candidate metabolites in the brain (D), hippocampus (E), and prefrontal cortex (F). Red and green bars denote the vote counting statistic for each candidate metabolite. An asterisk $\left(^{*}\right)$ indicates $p<0.05$. 5-HIAA 5-hydroxyindoleacetic acid, 5-HT serotonin, AMP adenosine monophosphate, LysoPC lysophosphatidylcholine, MG monoacylglycerol. 

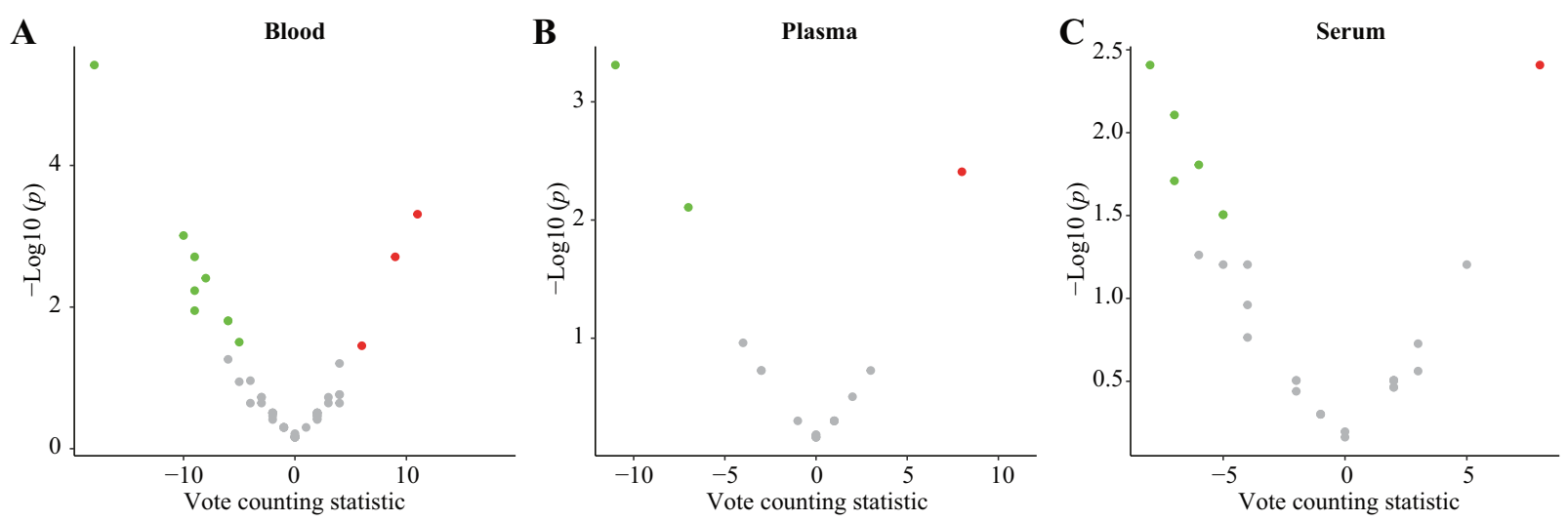

D

Blood

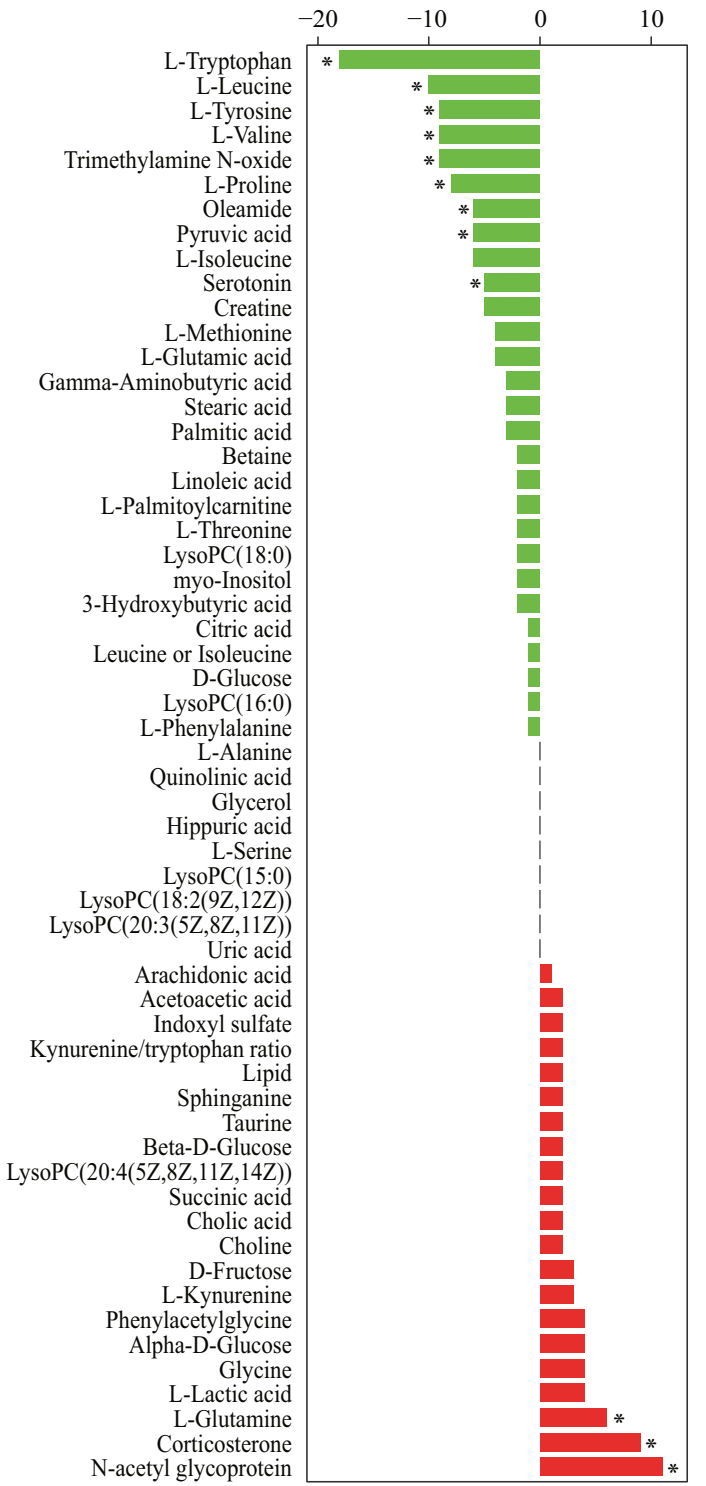

$\mathbf{E}$

Plasma

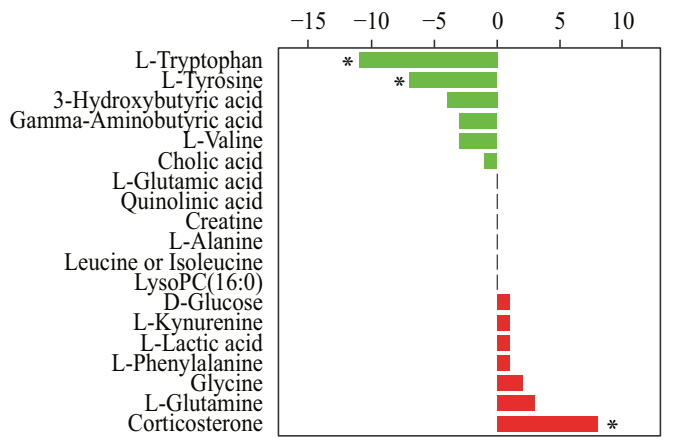

$\mathbf{F}$

Serum

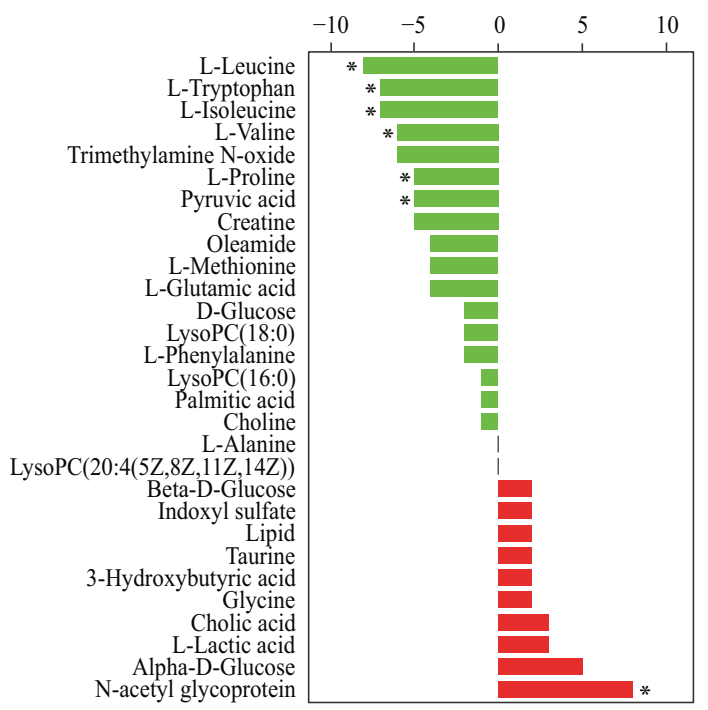

$\mathrm{N}$-acetyl glycoprotein

Fig. 3 Vote counting results for blood. A-C Volcano plots of candidate metabolites in blood (A), plasma (B), and serum (C). Nodes represent candidate metabolites, the $x$-axis shows the vote counting statistic, and the $y$-axis shows the $-\log 10$ ( $p$ value). Red, green, and gray nodes denote consistently upregulated, downregulated, or unaltered metabolites, respectively. D-F Bar plots of candidate metabolites in blood (D), plasma (E), and serum (F). Red and green bars denote the vote counting statistic for each candidate metabolite. An asterisk $\left(^{*}\right)$ indicates $p<$ 0.05. LysoPC lysophosphatidylcholine. 
A

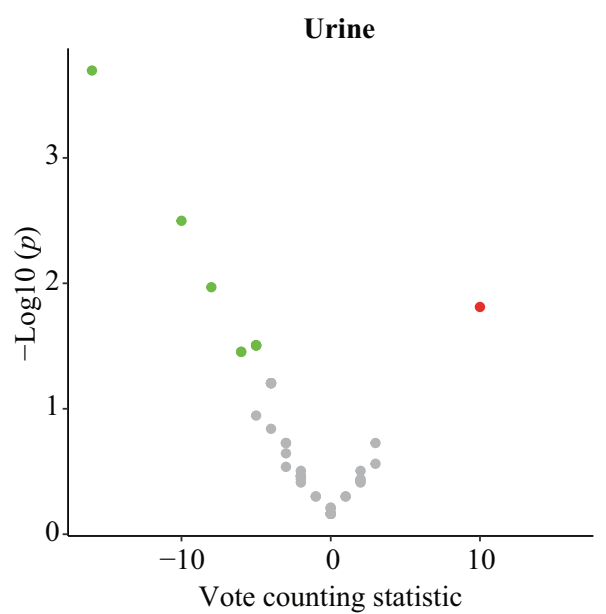

B

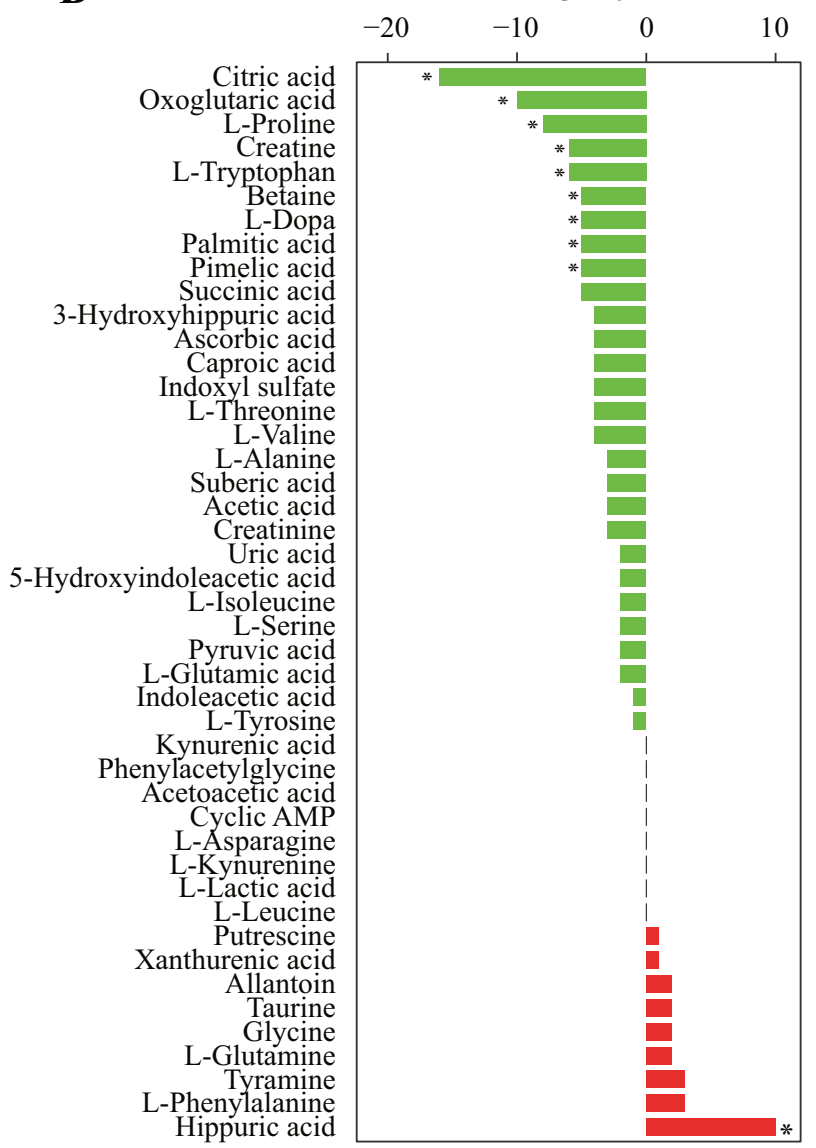

Fig. 4 Vote counting results in urine. A Volcano plots of candidate metabolites. Nodes represent candidate metabolites, the $x$-axis shows the vote counting statistic, and the $y$-axis shows the - log10 ( $p$ value). Red, green, and gray nodes denote consistently upregulated, downregulated, or unaltered metabolites, respectively. B Bar plots of candidate metabolites. Red and green bars denote the vote counting statistic for each candidate metabolite. An asterisk (*) indicates a $p<0.05$. AMP adenosine monophosphate.

Regarding the issue of result reproducibility, the heterogeneity of depression also needs to be considered. Our previous animal study found that different stress paradigms would lead to diverse patterns of metabolic changes in the hippocampus [29]. Clinical studies also revealed sex and age differences in the plasma metabolome signatures of MDD patients [30,31]. Despite these concerns, our systematic analysis suggested that certain metabolites were dysregulated across independent animal studies and expanded the understanding of the physiopathology of depression.

In the current study, we found that a variety of neurotransmitters were downregulated in the brains of depression models. Consistent with our expectations, we found that the central levels of monoamine neurotransmitters, including serotonin, dopamine, and norepinephrine, were decreased, supporting the classical monoamine hypothesis of depression [32]. We also found abnormalities in amino acid neurotransmitters, such as decreases in GABA levels in the brain and glutamic acid levels in the prefrontal cortex. These results are in agreement with the findings of previous meta-analyses, which showed lower brain GABA and glutamatergic metabolite levels in MDD patients [33-35]. These findings confirmed the role of altered glutamatergic and GABAergic neurotransmission in the pathophysiology of depression, as described in a previous review [36]. Another noteworthy observation is that we found downregulated anandamide levels in the brain. A relationship between anandamide, one of the main endocannabinoid metabolites, and the emotional state of animals was suggested. Previous studies found that decreasing anandamide levels by depletion of its producing enzyme may be a risk for the development of depression [37], and increasing its levels by blocking its hydrolase exerted antidepressant effects [38].

The results of this study showed upregulated kynurenine and hydroxykynurenine levels, as well as downregulated tryptophan levels in the brain. Kynurenine is synthesized from tryptophan and then broken down into hydroxykynurenine. The increased kynurenine to tryptophan ratio also suggested the activation of tryptophan metabolism to kynurenine by indoleamine 2,3dioxygenase [39]. Disturbances of kynurenine metabolites are associated with neuroimmune disturbance, as elevated kynurenine induced depression-like behaviors in rodents through monocyte trafficking and regulation of the NLRP2 inflammasome in astrocytes $[40,41]$. Therefore, this evidence highlighted that the neurotoxicity of kynurenine is a potential contributor to the pathogenesis of depression. Despite these findings, postmortem studies demonstrated unaltered in brain tissues of depressed individuals [42, 43], and previous meta-analyses reported decreased kynurenine levels and unaltered hydroxykynurenine concentrations in the blood of MDD patients [44, 45]. Therefore, further studies that determining the central levels of the kynurenine metabolites in MDD patients are still needed.

We found that the alterations in the blood of animal models were mainly characterized by decreases of amino acid concentrations. Two branched-chain amino acids (valine and leucine), two aromatic amino acids (tryptophan and tyrosine), and one other amino acid (proline) were downregulated. These results are in line with those of our recent meta-analysis, which showed a trend or statistical significance in decreases in these metabolites in the blood of MDD patients [22]. However, a decrease in the levels of these amino acids was not demonstrated to be a causal factor in the pathogenesis of depression, as depletion of these amino acids did not decrease mood in humans $[46,47]$. The possible reason for these findings is that stressed animals were in chronic catabolic states because of reduced food intake and weight loss, which may lead to disturbances in amino acid metabolism. In addition, we also identified upregulated glutamine levels. The association of circulating glutamine levels and depression is still controversial in clinical settings, as our previous meta-analysis reported decreased glutamine levels in MDD patients [22], while unaltered levels were reported in other meta-analyses [48, 49]. Therefore, further research on this topic is still needed.

Our results suggested upregulated corticosterone levels in the blood. Consistent with findings in MDD patients [50], increased circulating corticosterone supported the stress-induced hyperactivity of the hypothalamus-pituitary-adrenal axis, as previously 

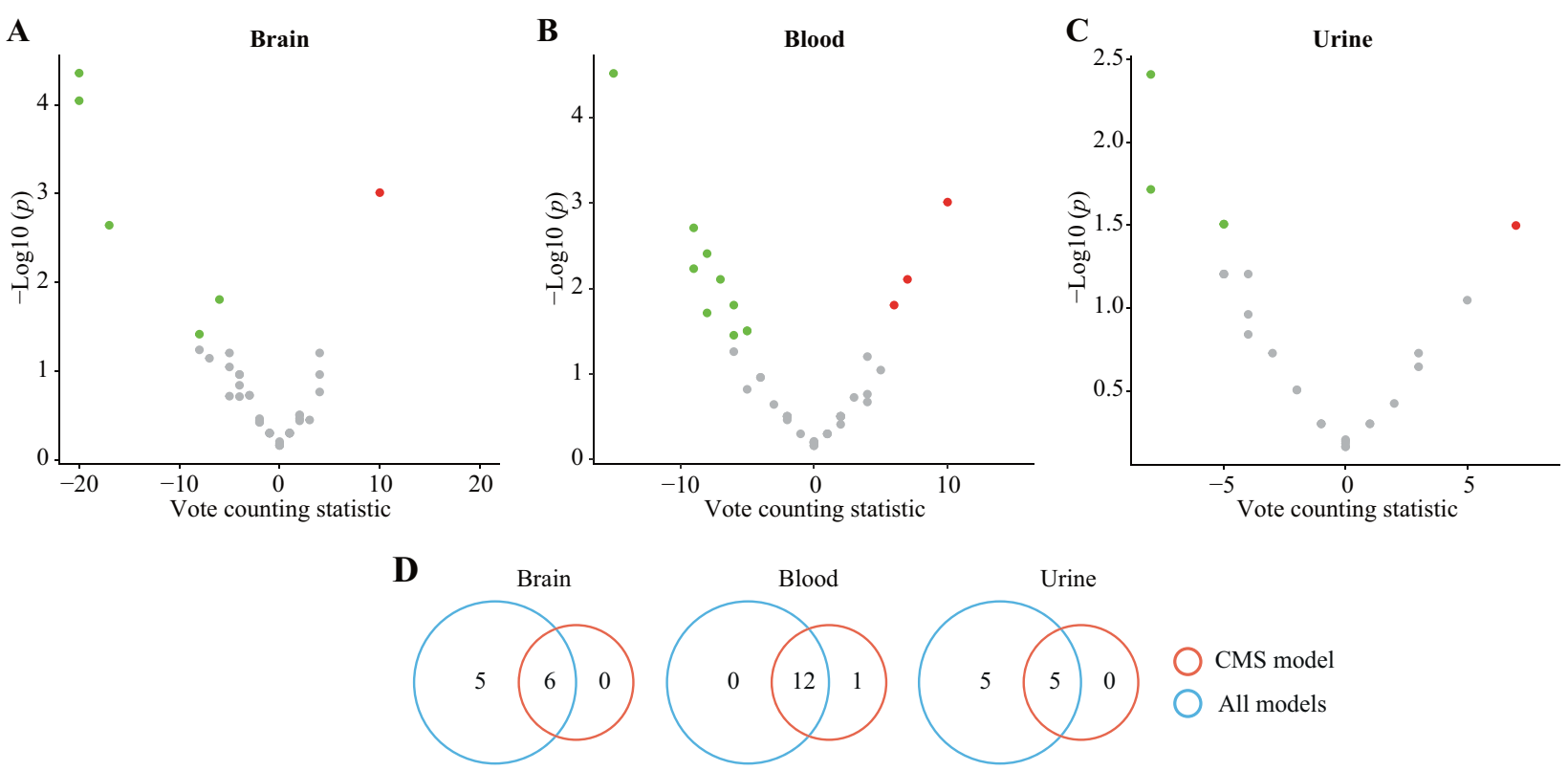

CMS model

All models

Fig. 5 Vote counting results in the chronic mild stress (CMS) model. A-C Volcano plots of candidate metabolites in the brain (A), blood (B), and urine $(\mathbf{C})$ of the CMS model. Nodes represent candidate metabolites, the $x$-axis shows the vote counting statistic, and the $y$-axis shows the $-\log 10$ ( $p$ value). Red, green, and gray nodes denote consistently upregulated, downregulated, or unaltered metabolites, respectively. D Venn diagram displaying the shared consistently dysregulated metabolites in all models and the CMS model.

discussed [51]. We also found that $\mathrm{N}$-acetyl glycoprotein was upregulated in the blood, which is consistent with data obtained in human studies [48]. N-Acetyl glycoprotein is a circulating marker of systemic inflammation because its concentration correlates with C-reactive protein, fibrinogen, and interleukin- 6 levels $[52,53]$. Therefore, these findings support that disturbances in the hypothalamus-pituitary-adrenal axis and peripheral inflammation are involved in the development of depression.

We found that three metabolites (citric acid, oxoglutaric acid, and creatine) involved in recycling of adenosine triphosphate (ATP) were downregulated in the urine of depression models. Moreover, pyruvic acid, an important metabolite in ATP metabolism, was also downregulated in the blood. These altered metabolites suggested an imbalance of energy metabolism in depression, which also could be explained by the chronic catabolic state. It was noted that supplementation of creatine or ATP resulted in antidepressant activity in preclinical studies $[54,55]$; therefore, energy metabolism may serve as a potential target for depression treatment.

Among the altered metabolites in urine, we also found that gut microbiota contributed to the physiopathology of depression. Hippuric acid was the only upregulated metabolite in urine, and its production in rats largely depends on the gut microflora [56]. We also identified downregulation of L-dopa, betaine, and tryptophan levels in urine and decreased trimethylamine $\mathrm{N}$-oxide levels in blood. The biochemical transformations of these metabolites involve a variety of gut microbiota, as described in a previous review [57]. Therefore, alterations in these microbial metabolites suggest the involvement of gut microbes in the development of depression, while further studies investigating the underlying pathogenic bacterium are still warranted.

Among the altered metabolites, tryptophan and serotonin were downregulated in both brain and peripheral tissues, and proline was downregulated in blood and urine samples. Moreover, some metabolites showed similar dysregulated trends in central and peripheral tissues. For example, tyrosine is the precursor of the monoamine neurotransmitters dopamine and epinephrine, its concentration was reduced in blood and also showed a downregulation trend in the brain and urine. In addition, it is also noticed an accumulation of kynurenine in the brain of depression models, with an increased trend in the blood. Central kynurenine is mainly synthesized from peripheral tryptophan degradation and then taken up into brain by transporters [58]. In MDD patients, plasma kynurenine levels correlated with its levels in the cerebrospinal fluid [59]. Other studies reported that attenuating the kynurenine accumulation in the brain by lowering plasma kynurenine levels or blocking the entry of kynurenine into the brain can exert an antidepressant effect $[60,61]$. Therefore, these findings highlight the bidirectional interactions of metabolomic changes between the brain and peripheral tissues.

Our study presents some limitations. First, application of the vote counting approach cannot identify new differential metabolites other than candidate metabolites. However, because only $3 \%$ of the included studies provided raw data, this method is still the most feasible way to perform such a large-scale quantitative analysis. To increase the accessibility of raw data, further studies with greater statistical power should be performed. Second, some metabolites were excluded from the semi-quantitative analysis because of an insufficient number of studies. Moreover, limited metabolite entries precluded stratified analyses. Future studies with more included studies are therefore recommended. Third, the metabolic alterations reported in this study may be caused by stress and are not specific to depression; therefore, further studies are needed to determine the causal relationship between these altered metabolites and the onset of depression. Finally, the publication bias was not examined as in an ordinary meta-analysis because of the use of vote counting.

In conclusion, we performed a quantitative analysis to identify consistently altered metabolites in the brain, blood, and urine of depression models based on 3743 differential metabolites from 241 metabolomics studies. The findings of this study suggested that metabolomic changes in depression models are characterized by decreased neurotransmitter and increased kynurenine metabolite levels in the brain, decreased amino acid and increased corticosterone levels in the blood, and imbalanced energy metabolism and microbial metabolites in urine. This study contributes to the existing knowledge of metabolomic changes 
in depression and reveals that the reproducibility of candidate metabolites was inadequate in previous studies.

\section{REFERENCES}

1. Hasin DS, Sarvet AL, Meyers JL, Saha TD, Ruan WJ, Stohl M, et al. Epidemiology of adult DSM-5 major depressive disorder and its specifiers in the United States. JAMA Psychiatry. 2018;75:336-46.

2. Huang Y, Wang Y, Wang H, Liu Z, Yu X, Yan J, et al. Prevalence of mental disorders in China: a cross-sectional epidemiological study. Lancet Psychiatry. 2019;6:211-24.

3. GBD 2017 Disease and Injury Incidence and Prevalence Collaborators. Global, regional, and national incidence, prevalence, and years lived with disability for 354 diseases and injuries for 195 countries and territories, 1990-2017: a systematic analysis for the Global Burden of Disease Study 2017. Lancet. 2018;392:1789-858.

4. Otte C, Gold SM, Penninx BW, Pariante CM, Etkin A, Fava M, et al. Major depressive disorder. Nat Rev Dis Prim. 2016;2:16065.

5. Geschwind DH, Flint J. Genetics and genomics of psychiatric disease. Science. 2015;349:1489-94.

6. Piazza I, Kochanowski K, Cappelletti V, Fuhrer T, Noor E, Sauer U, et al. A map of protein-metabolite interactions reveals principles of chemical communication. Cell. 2018;172:358-72.e23.

7. Medina CB, Mehrotra P, Arandjelovic S, Perry JSA, Guo Y, Morioka S, et al. Metabolites released from apoptotic cells act as tissue messengers. Nature. 2020;580:130-5.

8. Schrimpe-Rutledge AC, Codreanu SG, Sherrod SD, McLean JA. Untargeted metabolomics strategies-challenges and emerging directions. J Am Soc Mass Spectrom. 2016;27:1897-905.

9. Wishart DS. Metabolomics for investigating physiological and pathophysiological processes. Physiol Rev. 2019;99:1819-75.

10. Liu Y, Zhou X, Yang L, Wang H, Zhang Y, Pu J, et al. Social defeat stress causes depression-like behavior with metabolite changes in the prefrontal cortex of rats. PLOS ONE. 2017;12:e0176725.

11. Zhang $Y$, Yuan S, Pu J, Yang L, Zhou X, Liu L, et al. Integrated metabolomics and proteomics analysis of hippocampus in a rat model of depression. Neuroscience. 2018;371:207-20.

12. Prabhu VV, Nguyen TB, Cui Y, Oh YE, Piao YH, Baek HM, et al. Metabolite signature associated with stress susceptibility in socially defeated mice. Brain Res. 2019;1708:171-80.

13. Nasca C, Xenos D, Barone Y, Caruso A, Scaccianoce S, Matrisciano F, et al. L-acetylcarnitine causes rapid antidepressant effects through the epigenetic induction of mGlu2 receptors. Proc Natl Acad Sci USA. 2013;10:4804-9.

14. Locci A, Pinna G. Stimulation of peroxisome proliferator-activated receptor-a by $\mathrm{N}$-palmitoylethanolamine engages allopregnanolone biosynthesis to modulate emotional behavior. Biol Psychiatry. 2019;85:1036-45.

15. Zhang Y, Lu W, Wang Z, Zhang R, Xie Y, Guo S, et al. Reduced neuronal cAMP in the nucleus accumbens damages blood-brain barrier integrity and promotes stress vulnerability. Biol Psychiatry. 2020;87:526-37.

16. Duan J, Xie P. The potential for metabolomics in the study and treatment of major depressive disorder and related conditions. Expert Rev Proteom. 2020;17:309-22.

17. Pu J, Yu Y, Liu Y, Tian L, Gui S, Zhong $X$, et al. MENDA: a comprehensive curated resource of metabolic characterization in depression. Brief Bioinform. 2020;21:1455-64.

18. Wishart DS, Feunang YD, Marcu A, Guo AC, Liang K, Vázquez-Fresno $R$, et al. HMDB 4.0: the human metabolome database for 2018. Nucleic Acids Res. 2018;46:D608-17.

19. Kim S, Chen J, Cheng T, Gindulyte A, He J, He S, et al. PubChem in 2021: new data content and improved web interfaces. Nucleic Acids Res. 2021;49:D1388-95.

20. Kanehisa M, Furumichi M, Tanabe M, Sato Y, Morishima K. KEGG: new perspectives on genomes, pathways, diseases and drugs. Nucleic Acids Res. 2017;45: D353-61.

21. Fisher M, Feuerstein G, Howells DW, Hurn PD, Kent TA, Savitz SI, et al. Update of the stroke therapy academic industry roundtable preclinical recommendations. Stroke. 2009;40:2244-50.

22. Pu J, Liu Y, Zhang H, Tian L, Gui S, Yu Y, et al. An integrated meta-analysis of peripheral blood metabolites and biological functions in major depressive disorder. Mol Psychiatry. 2020. https://doi.org/10.1038/s41380-020-0645-4.

23. Guasch-Ferré $M$, Hruby A, Toledo $E$, Clish CB, Martínez-González MA, SalasSalvadó J, et al. Metabolomics in prediabetes and diabetes: a systematic review and meta-analysis. Diabetes Care. 2016;39:833-46.

24. Rikke BA, Wynes MW, Rozeboom LM, Barón AE, Hirsch FR. Independent validation test of the vote-counting strategy used to rank biomarkers from published studies. Biomark Med. 2015;9:751-61.
25. Goveia J, Pircher A, Conradi LC, Kalucka J, Lagani V, Dewerchin M, et al. Metaanalysis of clinical metabolic profiling studies in cancer: challenges and opportunities. EMBO Mol Med. 2016;8:1134-42.

26. Wang Y, Carter BD, Gapstur SM, McCullough ML, Gaudet MM, Stevens VL. Reproducibility of non-fasting plasma metabolomics measurements across processing delays. Metabolomics. 2018;14:129.

27. Lin Y, Caldwell GW, Li Y, Lang W, Masucci J. Inter-laboratory reproducibility of an untargeted metabolomics GC-MS assay for analysis of human plasma. Sci Rep. 2020;10:10918

28. Emwas AH, Roy R, McKay RT, Tenori L, Saccenti E, Gowda GAN, et al. NMR spectroscopy for metabolomics research. Metabolites. 2019;9:123.

29. Liu L, Zhou X, Zhang Y, Pu J, Yang L, Yuan S, et al. Hippocampal metabolic differences implicate distinctions between physical and psychological stress in four rat models of depression. Transl Psychiatry. 2018;8:4.

30. Pu J, Liu X, Liu Y, Gui S, Zhong X, Tian L, et al. Sex-specific plasma metabolome signatures in major depressive disorder. Psychiatry Clin Neurosci. 2019;73:713-4.

31. Zhou X, Liu L, Lan X, Cohen D, Zhang Y, Ravindran AV, et al. Polyunsaturated fatty acids metabolism, purine metabolism and inosine as potential independent diagnostic biomarkers for major depressive disorder in children and adolescents. Mol Psychiatry. 2019;24:1478-88.

32. Hirschfeld RM. History and evolution of the monoamine hypothesis of depression. J Clin Psychiatry. 2000;61 Suppl 6:4-6.

33. Schür RR, Draisma LW, Wijnen JP, Boks MP, Koevoets MG, Joëls $M$, et al. Brain GABA levels across psychiatric disorders: a systematic literature review and metaanalysis of (1) H-MRS studies. Hum Brain Mapp. 2016;37:3337-52.

34. Romeo B, Choucha W, Fossati P, Rotge JY. Meta-analysis of central and peripheral $Y$-aminobutyric acid levels in patients with unipolar and bipolar depression. J Psychiatry Neurosci. 2018;43:58-66.

35. Moriguchi S, Takamiya A, Noda Y, Horita N, Wada M, Tsugawa S, et al. Glutamatergic neurometabolite levels in major depressive disorder: a systematic review and meta-analysis of proton magnetic resonance spectroscopy studies. Mol Psychiatry. 2019;24:952-64.

36. Sanacora G, Treccani G, Popoli M. Towards a glutamate hypothesis of depression: an emerging frontier of neuropsychopharmacology for mood disorders. Neuropharmacology. 2012;62:63-77.

37. Lazary J, Eszlari N, Juhasz G, Bagdy G. Genetically reduced FAAH activity may be a risk for the development of anxiety and depression in persons with repetitive childhood trauma. Eur Neuropsychopharmacol. 2016;26:1020-8.

38. Carnevali L, Statello R, Vacondio F, Ferlenghi F, Spadoni G, Rivara S, et al. Antidepressant-like effects of pharmacological inhibition of FAAH activity in socially isolated female rats. Eur Neuropsychopharmacol. 2020;32:77-87.

39. Wang Y, Liu H, McKenzie G, Witting PK, Stasch JP, Hahn M, et al. Kynurenine is an endothelium-derived relaxing factor produced during inflammation. Nat Med. 2010;16:279-85.

40. Zang $X$, Zheng $X$, Hou $Y$, Hu M, Wang H, Bao X, et al. Regulation of proinflammatory monocyte activation by the kynurenine-AhR axis underlies immunometabolic control of depressive behavior in mice. FASEB J. 2018;32:1944-56.

41. Zhang Q, Sun Y, He Z, Xu Y, Li X, Ding J, et al. Kynurenine regulates NLRP2 inflammasome in astrocytes and its implications in depression. Brain Behav Immun. 2020;88:471-81.

42. Miller CL, Llenos IC, Dulay JR, Weis S. Upregulation of the initiating step of the kynurenine pathway in postmortem anterior cingulate cortex from individuals with schizophrenia and bipolar disorder. Brain Res. 2006;1073-1074:25-37.

43. Clark SM, Pocivavsek A, Nicholson JD, Notarangelo FM, Langenberg P, McMahon $\mathrm{RP}$, et al. Reduced kynurenine pathway metabolism and cytokine expression in the prefrontal cortex of depressed individuals. J Psychiatry Neurosci. 2016;41:386-94.

44. Marx W, McGuinness AJ, Rocks T, Ruusunen A, Cleminson J, Walker AJ, et al. The kynurenine pathway in major depressive disorder, bipolar disorder, and schizophrenia: a meta-analysis of 101 studies. Mol Psychiatry. 2020. https://doi.org/ 10.1038/s41380-020-00951-9.

45. Ogyu K, Kubo K, Noda Y, Iwata Y, Tsugawa S, Omura Y, et al. Kynurenine pathway in depression: a systematic review and meta-analysis. Neurosci Biobehav Rev. 2018;90:16-25.

46. Alhaj HA, Selman M, Jervis V, Rodgers J, Barton S, McAllister-Williams R. Effect of low-dose acute tryptophan depletion on the specificity of autobiographical memory in healthy subjects with a family history of depression. Psychopharmacology. 2012;222:285-92.

47. Roiser JP, McLean A, Ogilvie AD, Blackwell AD, Bamber DJ, Goodyer I, et al. The subjective and cognitive effects of acute phenylalanine and tyrosine depletion in patients recovered from depression. Neuropsychopharmacology. 2005;30:775-85.

48. Bot M, Milaneschi Y, Al-Shehri T, Amin N, Garmaeva S, Onderwater GLJ, et al Metabolomics profile in depression: a pooled analysis of 230 metabolic markers 
in 5283 cases with depression and 10,145 controls. Biol Psychiatry. 2020;87:409-18.

49. Huang T, Balasubramanian R, Yao Y, Clish CB, Shadyab AH, Liu B, et al. Associations of depression status with plasma levels of candidate lipid and amino acid metabolites: a meta-analysis of individual data from three independent samples of US postmenopausal women. Mol Psychiatry. 2020. https://doi.org/10.1038/ s41380-020-00870-9.

50. Carvalho AF, Köhler CA, Brunoni AR, Miskowiak KW, Herrmann N, Lanctôt KL, et al. Bias in peripheral depression biomarkers. Psychother Psychosom. 2016;85:81-90.

51. Pariante CM, Lightman SL. The HPA axis in major depression: classical theories and new developments. Trends Neurosci. 2008;31:464-8.

52. Lawler PR, Akinkuolie AO, Chandler PD, Moorthy MV, Vandenburgh MJ, Schaumberg DA, et al. Circulating N-linked glycoprotein acetyls and longitudinal mortality risk. Circ Res. 2016;118:1106-15.

53. Otvos JD, Shalaurova I, Wolak-Dinsmore J, Connelly MA, Mackey RH, Stein JH, et al. GlycA: a composite nuclear magnetic resonance biomarker of systemic inflammation. Clin Chem. 2015;61:714-23.

54. Cunha MP, Pazini FL, Lieberknecht V, Rodrigues ALS. Subchronic administration of creatine produces antidepressant-like effect by modulating hippocampal signaling pathway mediated by FNDC5/BDNF/Akt in mice. J Psychiatr Res. 2018;104:78-87.

55. Cao X, Li LP, Wang Q, Wu Q, Hu HH, Zhang M, et al. Astrocyte-derived ATP modulates depressive-like behaviors. Nat Med. 2013;19:773-7.

56. Gonthier MP, Verny MA, Besson C, Rémésy C, Scalbert A. Chlorogenic acid bioavailability largely depends on its metabolism by the gut microflora in rats. J Nutr. 2003;133:1853-9.

57. Krautkramer KA, Fan J, Bäckhed F. Gut microbial metabolites as multi-kingdom intermediates. Nat Rev Microbiol. 2021;19:77-94.

58. Cervenka I, Agudelo LZ, Ruas JL. Kynurenines: tryptophan's metabolites in exercise, inflammation, and mental health. Science. 2017;357:eaaf9794.

59. Haroon E, Welle JR, Woolwine BJ, Goldsmith DR, Baer W, Patel T, et al. Associations among peripheral and central kynurenine pathway metabolites and inflammation in depression. Neuropsychopharmacology. 2020;45:998-1007.

60. Agudelo LZ, Femenía T, Orhan F, Porsmyr-Palmertz M, Goiny M, MartinezRedondo $\mathrm{V}$, et al. Skeletal muscle PGC-1a1 modulates kynurenine metabolism and mediates resilience to stress-induced depression. Cell. 2014;159:33-45.

61. Walker AK, Wing EE, Banks WA, Dantzer R. Leucine competes with kynurenine for blood-to-brain transport and prevents lipopolysaccharide-induced depressionlike behavior in mice. Mol Psychiatry. 2019;24:1523-32.

\section{ACKNOWLEDGEMENTS}

This work was supported by the National Key Research and Development Program of China (Grant no. 2017YFA0505700), the Non-profit Central Research Institute Fund of
Chinese Academy of Medical Sciences (Grant no. 2019PT320002), and the Natural Science Foundation Project of China (Grant no. 81820108015 and 82101596).

\section{AUTHOR CONTRIBUTIONS}

Concept, design, and supervision of the study: PX, data acquisition/analysis/ interpretation: JP, YL, SG, LT, YY, XS, XZ, XC, WC, PZ, HZ, XG, LL, JW, and HW, drafting paper: JP, YL, and SG, drafting display items: JP. All authors read and approved the final version of the paper.

\section{COMPETING INTERESTS}

The authors declare no competing interests.

\section{ADDITIONAL INFORMATION}

Supplementary information The online version contains supplementary material available at https://doi.org/10.1038/s41380-021-01269-w.

Correspondence and requests for materials should be addressed to P.X

Reprints and permission information is available at http://www.nature.com/ reprints

Publisher's note Springer Nature remains neutral with regard to jurisdictional claims in published maps and institutional affiliations.

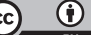

Open Access This article is licensed under a Creative Commons Attribution 4.0 International License, which permits use, sharing, adaptation, distribution and reproduction in any medium or format, as long as you give appropriate credit to the original author(s) and the source, provide a link to the Creative Commons licence, and indicate if changes were made. The images or other third party material in this article are included in the article's Creative Commons licence, unless indicated otherwise in a credit line to the material. If material is not included in the article's Creative Commons licence and your intended use is not permitted by statutory regulation or exceeds the permitted use, you will need to obtain permission directly from the copyright holder. To view a copy of this licence, visit http://creativecommons. org/licenses/by/4.0/

(c) The Author(s) 2021 